Article

\title{
X-Ray Photon Correlation Spectroscopy with Coherent Nanobeams: A Numerical Study
}

\author{
Oier Bikondoa ${ }^{1,2, *(\mathbb{D})}$ and Dina Carbone ${ }^{3, *(\mathbb{D})}$ \\ 1 Department of Physics, University of Warwick, Gibbet Hill Road, Coventry CV4 7AL, UK \\ 2 XMaS, The UK Materials Science Facility, The European Synchrotron Radiation Facility, CS40220, \\ F-38043 Grenoble CEDEX, France \\ 3 MAX IV Laboratory, Fotongatan 2, 22592 Lund, Sweden \\ * Correspondence: oier.bikondoa@esrf.fr (O.B.); gerardina.carbone@maxiv.lu.se (D.C.)
}

Received: 18 July 2020; Accepted: 26 August 2020; Published: 29 August 2020

\begin{abstract}
X-ray photon correlation spectroscopy accesses a wide variety of dynamic phenomena at the nanoscale by studying the temporal correlations among photons that are scattered by a material in dynamical equilibrium when it is illuminated with a coherent X-ray beam. The information that is obtained is averaged over the illuminated area, which is generally of the order of several square microns. We propose here that more local information can be obtained by using nanobeams with great potential for the study of heterogeneous systems and show the feasibility of this approach with the support of numerical simulations.
\end{abstract}

Keywords: coherence; X-rays; XPCS; nanobeams; diffraction limited sources, numerical study

\section{Introduction}

Recent developments of synchrotron sources pursue the drastic reduction of the source emittance and the increase of brilliance, which translates into a huge gain in the coherent photon flux at the sample [1]. One of the experimental approaches that takes full advantage of this gain is X-ray photon correlation spectroscopy (XPCS), a well established technique that provides access to the dynamics of nano- to micro-scale materials in a wide range of time-scales, from hundreds of seconds to sub- $\mu s$ [2-10], and has recently been demonstrated at the $n s$ scale [11]. Derived from the Dynamical Light Scattering technique (DLS) [12-14], which uses coherent light from laser sources, XPCS exploits the coherence properties of X-ray beams produced at 3rd and 4th generation sources to inspect time-resolved interference patterns, also called "speckles", from disordered samples. The fluctuation of speckles in time, arising from the changing sample configuration, are analysed in terms of time correlation functions. These give direct access to the characteristic times of the probed dynamics that can be related to diffusion, relaxation, coalescence, phase transitions, etc. XPCS is complementary to DLS and the time domain accessible with both techniques is similar [2,15]. However, while DLS, due to the nature of the probe, is limited to the study of length scales $>100 \mathrm{~nm}$, XPCS can explore length scales that range from nanometers to atomic distances [16]. In addition, it can be used to study systems that are opaque to visible light and is not affected by multiple scattering effects $[2,16]$. The capability of acquiring data at high time rate is a fundamental requirement for this technique, the fastest dynamics accessible being limited by the shortest acquisition time achievable during the experiment. This, along with the demand of high coherence, makes the number of scattered coherent photons per unit time the parameter of largest relevance for an XPCS experiment-provided, certainly, that the acquisition rate of the available detectors is not a bottle-neck-which in many cases is indeed the case. Needless to say, stability of the whole setup, from the X-ray source to the detector, including optics and sample environment, over all the measurement time is a conditio sine qua non. 
The field of application of XPCS has originally developed around the investigation of equilibrium dynamics of meso-scale particle assemblies as colloids, gels and polymers $[2,15]$ studied with small angle scattering geometry (SAXS) and it has later extended towards out-of-equilibrium systems, such as complex fluids and rheology $[17,18]$ or ageing in self-organised systems $[19,20]$. The access to increased coherent flux has opened the field to include atomic fluctuations or diffusion in hard-condensed matter systems, such as ageing and phase transitions in metallic glasses [6,21], diffusion in alloys [22-24], order-disorder fluctuations in crystals close to phase transitions [25] and even flow and diffusion of "phase discontinuities" as defects in thin metal films [26] (see also [8] and references therein). XPCS approaches have also been applied to the study of fluctuation of domains in magnetic [27-30] and ferroelectric [31] materials, and to the study of charge density waves under current [32]. For many of the mentioned systems, the relevant signal consists of diffuse scattering in the vicinity of Bragg peaks, with intensity typically orders of magnitudes weaker, see for example [31] where the measured intensity is $\sim 10^{-3} \mathrm{ph} / \mathrm{s} /$ pixel. For other systems, like magnetic materials, the scattering cross-section, even exploiting enhancement effects at resonance energies [30], is smaller than the charge scattering by 3-4 orders of magnitude. This loss of intensity in the speckles limits strongly the accessible range of time-scales for these studies. Therefore, in many reported cases, the system dynamics and characteristic times have to be tuned (namely slowed down) with external parameters, like temperature, external magnetic or electric field, applied currents, etc. for the benefit of the counting statistics of the experiment performed. Repeating or extending the measurement time is also used to increase signal statistics, when this is not in conflict with the intrinsic aging of the system and does not determine a loss of information of the system behaviour. Therefore, any further development of the XPCS technique towards the investigation of these material systems at the relevant time-scales relies heavily on the access to increased coherent flux, along with the capability of adjusting the set-up to match the requirements of each experiment. Free Electron Lasers-FELs offer in principle the ultimate increase in coherent flux and time resolution for XPCS. As an example, the XPCS dedicated beamline of LCLS source can provide a coherent flux of $\sim 10^{10} \mathrm{ph} / \mathrm{s}$, in monochromatic mode in the energy range $5-10 \mathrm{keV}$-equivalent to $\sim 10^{12}$ photons per pulse at $\sim 10^{-4}$ monochromator bandwidth and repetition rates of $120 \mathrm{~Hz}$, and a time-resolution in the ms-scale [33]. However, beam jittering in energy, intensity and position still represent a strong limiting factor and lots of effort is put into technological and methodological developments to overcome these issues and make full use of the exceptional coherence and time-resolution of these sources [11]. Due to the intrinsic stability of the X-ray production process, synchrotrons are arguably the best sources to perform XPCS.

We propose here that the use of nanobeams for XPCS (nXPCS) combines the benefits of a stable X-ray source and a dramatic increase of coherent flux, with the added value of a reduction of the size of the illuminated volume that introduces a nanometre-range lateral resolution until now unexploited. In this manuscript, we discuss how the reduction of the beam size, by using nano-focusing optics, allows access to local information at the nanoscale, with excellent spatial resolution, and how this, combined with lateral translation of the sample in the beam, benefits the study of spatially heterogeneous systems. We demonstrate the power of using nanobeams with the support of simulations, awaiting experimental setups to match the appropriated-scaled, stability levels. We note here that for beam sizes comparable with particles size or inter-particle distances, this approach requires a modification of the standard theoretical framework to account for particle number fluctuations within the volume [34]. Here, we stay far from these limits, and concentrate onto the conditions that will get all the benefits of decreasing the beam size, while preserving the applicability of the standard XPCS analysis.

\section{XPCS Theory}

We include here a brief theoretical description of the mathematical background for the XPCS analysis. Comprehensive introductions and relevant references can be found in Ref. [2,5,7]. In conventional XPCS experiments, a monochromatic and coherent $\mathrm{X}$-ray beam impinges on a sample and the scattered 
signal—speckle pattern [35]—is recorded on a detector placed in the far field. Speckles appear due to the propagation of the $\mathrm{X}$-ray beam through a medium with random fluctuations of the electronic density or by the reflection from a rough surface. In a dynamical medium where spontaneous electronic density fluctuations occur in time (either by changes of the electronic density or movements of the constituting particles of the medium), the intensity of the speckles will also fluctuate. In XPCS, speckle intensity correlation functions are employed to obtain information about the dynamics of the system. $g^{(2)}(\mathbf{Q}, \tau)$, the normalised intensity correlation function of the speckles is defined as:

$$
g^{(2)}(\mathbf{Q}, \tau)=\frac{\left\langle I_{t} I_{t+\tau}\right\rangle_{\mathrm{T}}}{\left\langle I_{t}\right\rangle_{\mathrm{T}}^{2}}=\frac{\left\langle E_{t}^{*} E_{t+\tau}^{*} E_{t} E_{t+\tau}\right\rangle_{\mathrm{T}}}{\left\langle\left|E_{t}\right|^{2}\right\rangle_{\mathrm{T}}^{2}}
$$

The superscript ${ }^{(2)}$ indicates that $g^{(2)}$ is a temporal correlation function of order two [36]. $I_{t}$ is short for $I(\mathbf{Q}, t)$, the intensity at time $t$ and at momentum transfer $\mathbf{Q} . \tau$ is the time delay. The angle brackets indicate an average over the acquisition time $\mathrm{T}$.

The Siegert relation connects $g^{(2)}(\mathbf{Q}, \tau)$ and the intermediate scattering function (ISF) of the sample $S(\mathbf{Q}, \tau)[13,37]$ :

$$
g^{(2)}(\mathbf{Q}, \tau)=1+\beta(\mathbf{Q})\left[\frac{S(\mathbf{Q}, \tau)}{S(\mathbf{Q})}\right]^{2}
$$

The optical contrast $\beta(\mathbf{Q})=\frac{\sigma^{2}}{\langle I\rangle}$ is a factor that is used to account for the degree of spatial coherence of the incident radiation and is given by the variance of the intensity $\left(\sigma^{2}\right)$ divided by its mean value [38]. $S(\mathbf{Q})$ is the static structure factor. The $\operatorname{ISF}(S(\mathbf{Q}, \tau))$ is the spatial Fourier transform of the density correlation function and the time Fourier transform of the dynamical structure factor [39]. The time dependence of the electronic density correlations in the sample can be deduced from the ISF. However, this is usually a very complicated function to get. Equation (2) is not general: it is valid if the range of spatial correlations in the system is much smaller than the linear dimension of the scattering volume [13]. In that case, the central limit theorem of statistics can be applied and all the correlation functions can be expressed in terms of the lowest order ones [40]. However, when nanobeams are used, it is rather likely that the spatial correlations are of the order of the illuminated volume and that a straightforward association between $g^{(2)}(\mathbf{Q}, \tau)$ and the ISF through the Siegert relation should be abandoned. Nevertheless, one can always resort to using simulations to relate the ISF and the intensity fluctuations. For the results presented here, the spatial correlations are smaller than the illuminated volume.

\section{Experimental Considerations}

The speckle intensity determines the shortest and the longest time-scales accessible during the experiment. For samples with low scattering power (i.e., small volume and/or scattering cross-section), the total measurement time $T$ needed to acquire enough statistics can be up to orders of magnitude larger than the accumulation time $\tau_{a}$. One way to improve statistic is by acquiring simultaneously a large number of speckles $N_{s p}$, which is routinely done by using 2D pixel detectors. These mentioned quantities contribute to the figure of merit used to estimate XPCS data quality, i.e., the signal-to-noise ratio $s / n$ defined as [41]

$$
\frac{s}{n}=I_{0} \beta \sqrt{\tau_{a} T N_{s p}}
$$

where $I_{0}$ and $\beta$ represent the intensity of the incoming beam and the fraction of coherent beam (in \%), respectively. The need of an intense coherent radiation explains why this technique has been developed at synchrotron sources and is now pursued also at FEL facilities. The coherence properties of the X-ray beam produced are often expressed in terms of transverse (or lateral) and longitudinal (or temporal) coherence lengths, respectively $\zeta_{r}$ (with $r=x, y$ in the plane of propagation of the X-ray beam) and 
$\zeta_{t}$, which depend on the wavelength used $(\lambda)$, the size of the source along the relevant direction $\left(\Sigma_{r}\right)$, and its distance $(d)$ from the measurement point in the following way:

$$
\zeta_{r}=\frac{\lambda d}{2 \Sigma_{r}}, \quad \zeta_{t}=\frac{\lambda^{2}}{\Delta \lambda}
$$

Dedicated beamlines at 3rd generation synchrotron sources typically provide a fully coherent photon flux of the order of $1-5 \cdot 10^{8} \mathrm{ph} / \mathrm{s}$ in the photon energy range of $6-15 \mathrm{keV}$, and transverse coherent lengths of the order of few to few tens microns. The temporal coherence length depends on the monochromaticity of the X-ray beam $\Delta \lambda / \lambda$, typically of the order of $10^{-4}$ with a standard $\operatorname{Si}(111)$ monochromator. Slits or pinholes are generally used to reduce the lateral size of the X-ray beam to match its transverse coherent lengths, so that the conditions of coherent illumination of the sample are satisfied. Because XPCS is based on the visibility of the speckles and is therefore quite forgiving on small compromises on the degree of coherence of the X-ray beam, a further increase of intensity can be obtained by using larger pinholes and accepting larger portions of the incoming beam. The increased brilliance of the 4th generation Diffraction Limited Storage Rings (DLSR) is expected to result in up to 100-fold increase of coherent flux in the same energy range, when combining the increase of flux density due to the reduction of the beam divergence, and the increase of transverse coherence length due to a reduction of the source size $\Sigma_{r}$ (cf. Equation (4)). From Equation (3), this can result in a $100^{2}$-fold decrease of $\tau_{a}$ with an obvious benefit for the study of systems with faster dynamics. However, this increase of the size of the coherent beam has a strong impact on the experimental setup. Like all coherent scattering experiments, the intensity speckles measured in XPCS are well described by the square of the Fourier transform of the electronic distribution of the illuminated sample-multiplied by the complex illumination function, at each measurement time. This results in a relation of inverse proportionality between coherent beam size $\zeta_{r}$ and speckle size $w_{r}$ measured at a distance $D$ :

$$
w_{r}=\frac{D \lambda}{\zeta_{r}} .
$$

The detectors most used for XPCS measurements are photon-counting pixel detectors, which nowadays can provide pixels of the order of $50-100 \mu \mathrm{m}$ in size and acquisition rates of the order of few $\mathrm{kHz}$ (up to $22 \mathrm{kHz}$ if operated in burst mode [42]). It is widely accepted that a detector pixel matching the size of the speckle is the most efficient way of measuring XPCS data [43]. It results, from Equation (5), that setups typically developed for XPCS in the hard X-ray regime, with $\lambda \sim 10^{-10} \mathrm{~m}$, are rather bulky and include sample-detector distances of the order of several metres. The beams produced at DLSR and FELs provide an even larger transverse coherence length, which pushes further the demands on the setup. Moreover, because the information obtained in XPCS experiments is ensemble averaged over the illuminated volume, the use of larger beams, while certainly benefiting the measurement statistics of laterally uniform systems, is disadvantageous for systems confined in small spaces (i.e., small volumes) and for those with intrinsic lateral heterogeneity. X-ray nanobeams are routinely produced at dedicated beamlines, with lateral dimensions ranging from few tens to a couple of hundred nanometres. Therefore, nXPCS experiments can overcome both the above-mentioned issues, by keeping the needed sample-detector distance within one metre and allowing an efficient measurement of systems confined in small volumes, with the further benefit for the study of heterogeneous samples.

\section{Simulations}

In order to show the effect of using a small beam, we have carried out simulations using 2D matrices with particles in Brownian motion, which is a rather typical model system for XPCS. First, we study three different but interrelated model systems and their results together (Figure 1): 
- Model 1 (M1) consists of a number $N_{1}$ of particles in Brownian motion with diffusion constant $\eta_{1}$, unconfined, i.e., free to move within the whole matrix (Figure 1, left inset). This represents the ideal ergodic system for XPCS analysis.

- Model 2 (M2) consists of two types of particles in Brownian motion with same shape: $N_{1}$ particles with diffusion constants $\eta_{1}$ and $N_{2}$ with diffusion coefficient $\eta_{2}$, both unconfined (Figure 1 , middle inset). It is an elementary representation of a system with heterogeneous dynamics but which is spatially homogeneous.

- Model 3 (M3) contains two types of particles, as for M2, but at time $t=0$, the $N_{2}$ particles are confined in a small region in the center of the box (Figure 1, right inset). In other words, at time $t=0$, the central region has a high concentration of slow particles that are free to move. This model is a simple representation of a spatially heterogeneous system or a confined system because, for the total time used in our simulations, the slow particles stay in a region close to the matrix centre. It is clearly a non-ergodic system.
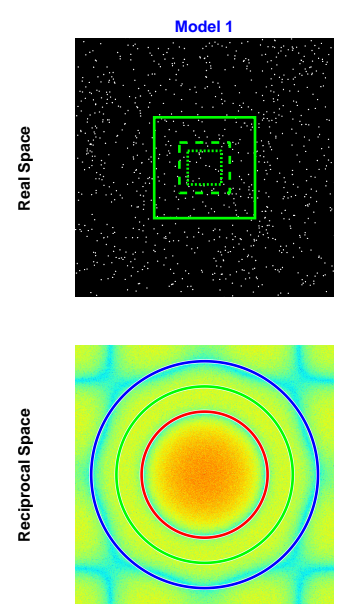
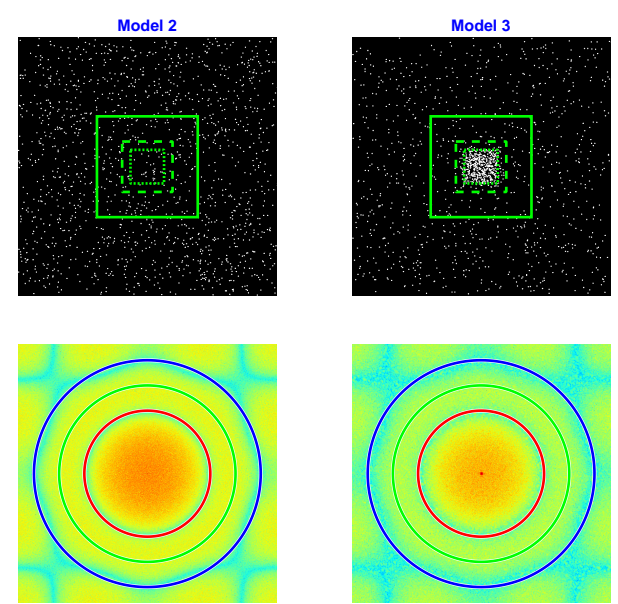

Figure 1. Matrices representing the real space configuration (top row) and the reciprocal space speckles (bottom row), in logarithmic scale, of the three different models in the initial conditions: (left) Real space representation of Model 1, where one type of particle is uniformly distributed in the 2D matrix at time $\mathrm{t}=0$; (middle) Model 2: two types of particles of same shape and different diffusion constant are uniformly distributed in the matrix; (right) Model 3: one type of particle is uniformly distributed in the 2D matrix and and a second type of slower particles are confined (in time $t=0$ ) in a small area in the middle of the matrix. The green squares on the top row images represent the different mask sizes that have been applied (masks 1, 2 and 3 in the main text). The circles on the bottom row figures indicate the distances (in reciprocal space) at which the OTCFs have been extracted $(250,350, \& 450$ in the main text-These correspond respectively to $Q$ values of $0.11,0.15$, and $0.20 \mathrm{~nm}^{-1}$ for a particle of $80 \mathrm{~nm}$ diameter).

A fourth model (M4), where three types of particles in Brownian motion and different diffusion constants are spatially separated at time $t=0$, will be presented in Section 4.3.

\section{Simulation Details}

Each model is represented, for simplicity, by a 2D matrix of dimension $N \times N$ which corresponds to the projection of a 3D volume along the beam direction. In analogy, spherical particles are approximated in 2D by a circular shape. For M1 \& M2, the distribution of particles is random and homogeneous in the 2D matrix, while for M3 the $N_{2}$ particles with slower dynamics $\left(\eta_{2}=0.1 \times \eta_{1}\right)$ are initially concentrated in a $128 \times 128$ pixel area (Figure 1 ). For the simulations shown here, it is $N=1024, N_{1}=1024$ and $N_{2}=512$. Round particles were assigned to each position, approximated by $5 \times 5$ pixels with the four corner pixels removed. The pixel values of all particles are set to 1 . Using other shapes did not alter the results. The same shape was selected for all types of particles and 
the dispersity index for each type of particle is set to 0 to shun any artifact arising from different form factors and that might be echoed on the correlation functions. For each model, four XPCS synthetic data-sets have been produced, simulating different illumination conditions of the systems: the first corresponding to a large beam, where the whole 2D matrix is contributing to the scattering signal, and the other three with beams of progressively reduced size (masks 1, $2 \& 3$, green squares in Figure 1).

The simulations were done using MATLAB [44]. Trajectories with 500 points for a total time of $10 \mathrm{~s}$, starting from the origin and following Brownian motion were created for a number $N_{p}$ of particles, with $N_{p}=N_{1}+N_{2}$ for M2 \& 3 and $N_{p}=N_{1}$ for M1. Each individual trajectory was calculated using J. Burkardt's GNU-LGPL licensed code [45]. A normally distributed spatial step size $s=2 d \eta \Delta t$, where $d$ is the dimensionality ( $d=2$ in our simulations), $\eta$ the diffusion constant and $\Delta t$ time step ( $\Delta t=$ total time/number of points) was used. Random starting points were then selected for the particles 1 (2) within an area $A_{1}\left(A_{2}\right)$ inside a $N \times N$ grid according to the requirement of each model (cf. Table 1 ), and each trajectory was shifted to these starting positions.

Snapshots of the real space $N \times N$ grid were calculated for each time step. Three different masks were subsequently applied to each snapshot to simulate the use of nanobeams of different sizes, and the illumination of progressively smaller portions of the sample (see Figure 1, top). The masks consisted of a $N \times N$ matrix with pixels set to zero everywhere but in a central $M \times M$ area, with $M=400,200$, 133 for masks $1,2 \& 3$, respectively. The size of the masks was chosen to progressively reduce the illuminated portion towards the area of the "confined" sample $A_{2}$. This way of proceeding allows one to use the same trajectories for the different masks and study the sole effect of reducing the beam size on the synthetic data-sets. The masked snapshots were subsequently fast-Fourier transformed and squared to calculate the intensity distribution in reciprocal space (Figure 1, bottom). This operation implies a full coherence of the incoming beam. Besides this, no specific assumptions are made about the X-ray energy or the intensity of the impinging beam, and no noise was added to the dataset obtained, as our study is not concerned about the quality of the signal, and assumes that the scattered intensity is enough to provide a good signal-to-noise ratio.

All the parameters used for the simulations are summarised in Table 1. To give a measure of the corresponding experimental conditions in a real-life experiment, for a particle size of $30 \mathrm{~nm}$, our simulations correspond to a full beam of lateral size $6 \times 6 \mu \mathrm{m}^{2}$ and to beams of lateral dimensions of $2.4 \times 2.4 \mu \mathrm{m}^{2}, 1.2 \times 1.2 \mu \mathrm{m}^{2}$ and $800 \times 800 \mathrm{~nm}^{2}$, respectively, for the masks $1,2 \& 3$. The corresponding increase in flux density is a factor of 6,23 and 53 assuming an efficiency of the focusing optics of $90 \%$, a realistic value for KB mirror optics. The flux increase corresponds to $0.6,2.6$ and 6 times, for a focusing optics efficiency of 10\%, typical value for Fresnel Zone Plates in the hard X-ray regime, as an example. As we use 2D matrices for the simulations, it is more adequate to consider an area fraction $(\Phi)$ instead of a volume fraction. In M1, M2 and M3, $\Phi_{1} \sim 0.02$, and in $\mathrm{M} 2 \& \mathrm{M} 3, \Phi_{2} \sim 0.02$. In M3, the $N_{2}$ particles are concentrated or confined in a small region in the centre. If only that region is taken as the area, then $\Phi_{2} \sim 1$.3. However, we note that our 2D matrix is a projection of the scattering volume along the beam direction, so this apparently unrealistic value, translated into a volume fraction in a $3 \mathrm{D}$ experiment is $\Phi_{2} \sim 1.3 / 128 \sim 0.01$.

One time correlation functions (OTCF) were calculated for each pixel and averaged for all the pixels with equivalent $Q$-value (circles in Figure 1, bottom). These correspond respectively to $Q$ values of $0.11,0.15$ and $0.20 \mathrm{~nm}^{-1}$ for a particle of $80 \mathrm{~nm}$ diameter. In the rest of the manuscript, these values are referred to as "distance" measured in pixels from the center of the intensity matrix. The time evolution of the four models and the simulation of XPCS data in the different conditions are detailed in Section 4. For M4, we followed the same procedure except that the mask size was fixed to $(101 \times 101)$ pixels and its position was displaced along one of the diagonals of the matrix, to give the clearest graphical representation of the raster scan. Rastering the beam horizontally on M4 yields the same results. 
Table 1. Simulation parameters for models $1,2 \& 3$ and for the masks. Nomenclature, with subscripts 1 and 2 indicating fast and slow particles, respectively: $N_{1,2}$ : number of particles; $A_{1,2}$ : initial area (in pixels) on which the particles are positioned; $\eta_{1,2}$ : diffusion constant.

\begin{tabular}{cccc}
\hline & Model 1 & Model 2 & Model 3 \\
\hline$N_{1}$ & 1024 & 1024 & 1024 \\
$A_{1}$ & $1024 \times 1024$ & $1024 \times 1024$ & $1024 \times 1024$ \\
$\eta_{1}$ & 64 & 64 & 64 \\
$N_{2}$ & - & 1024 & 1024 \\
$A_{2}$ & - & $1024 \times 1024$ & $128 \times 128$ \\
$\eta_{2}$ & - & $0.1 \eta_{1}$ & $0.1 \eta_{1}$ \\
\hline & Mask 1 & Mask 2 & Mask 3 \\
\hline$M$ & 400 & 200 & 133 \\
\hline
\end{tabular}

\section{Results}

\subsection{Homogeneous vs. Heterogeneous Systems}

By comparing the OTCFs of the three models M1, M2 \& M3 using different beam sizes (Figure 2), we observe that, in the case of the two spatially homogeneous systems (M1 \& M2), the reduction in beam size does not change the one-time correlation functions appreciably: when reducing the beam size-provided that the beam size is not so small that number fluctuations become significant (see [34] and the discussion) — the particles' relative contributions to the scattered signal decrease by the same amount. This is a manifest consequence of the uniformity of these systems, which, observed at different spatial scales-but still large in comparison with the particle size-look alike. In contrast, for the spatially heterogeneous system M3, the measured correlation function shows a strong dependence on the beam size and the illuminated area.
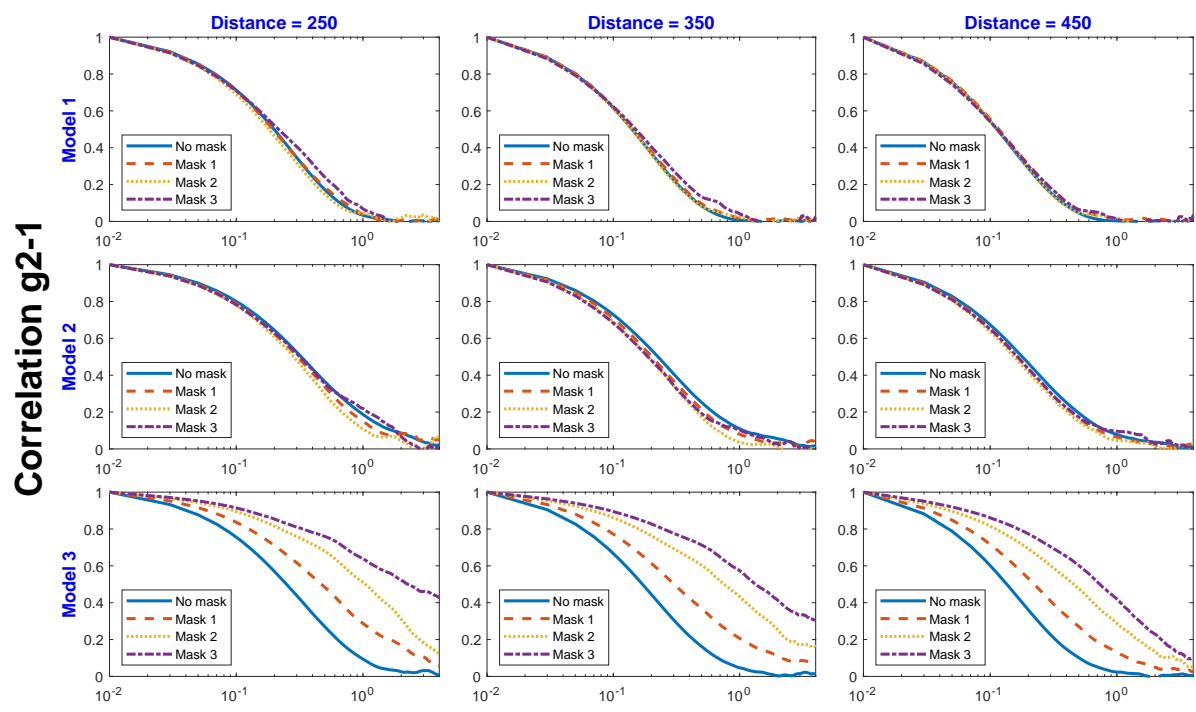

Time [arb. units]

Figure 2. One-time correlation functions for the different models at distances 250, 350 \& 450, for different masks. This representation stresses the effect of adding masks for each model.

Interestingly, and rather obviously too, for a heterogeneous system, there is also a dependence on the decaying times with the beam size. This can be observed in Figure 3, where the OTCFs of the three models are plotted together again, but for different masks (beam sizes) and distances. When a large beam is used (Figure 3, top row), the OTCFs and thus the time constants that are obtained for each model show only subtle differences. By progressively reducing the beam size (Figure 3 from top to bottom row), the results stay the same for M1 \& M2, while changing dramatically for the heterogeneous 
system M3: the small beam size increases sensitivity to the slow moving particles by increasing their relative contribution with respect to the fast moving ones. We can therefore posit that large beams are rather insensitive to spatial heterogeneities and that one needs beams that are comparable to the size of the heterogeneities to examine their dynamical behaviour.
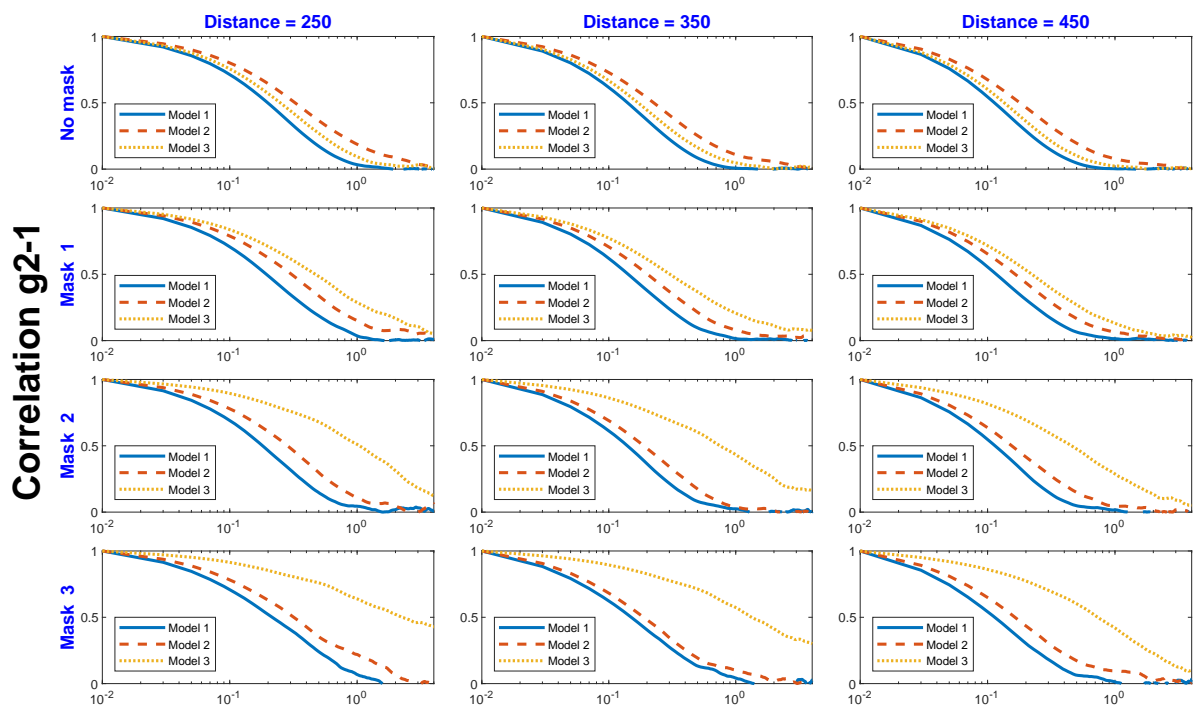

Time [arb. units]

Figure 3. Equivalent representation to that of Figure 2 of the one-time correlation functions for the different models at distances 250, $350 \& 450$, for different masks. This representation stresses the differences in the OTCFs that are obtained for the different models.

\subsection{Particle Concentration in a Heterogeneous System}

To investigate the possible influence of particle concentration on the heterogeneous system M3, we have performed simulations with three different concentrations of slow moving particles (Figure 4) and apply the same masks as in Figure 3.

When the full beam is used (no mask: Figure 4, topmost row), similar OTCFs are obtained with the three concentrations. If the beam size is reduced, the OTCFs deviate gradually (Figure 4, going from top to bottom row). We have extracted the the decay rates of each OTCF in Figure 4, at distance 450 (right column) by fitting the curves to a Kohlrausch-Williams-Watts (KWW) function of the form $f(t) \propto \exp -(\Gamma t)^{\gamma}$, with $\Gamma$ and $\gamma$ being the decay rate and the KWW exponent in the decay function, respectively. $\gamma>1(\gamma<1)$ implies that the decay is faster (slower) than purely exponential. The KWW function appears frequently in XPCS studies of non-equilibrium systems and heterogeneous dynamics [38]. The decay rates for the different concentrations vs. mask size (Figure 5) have a similar trend and decrease when the beam size is reduced because the relative concentration of slow moving particles with respect to the fast moving ones in the illuminated volume is enhanced. The decay rate is also slower for higher concentrations of slow particles, as foreseeable. Still, the behaviour of the KWW exponent does not follow such a systematic trend (Figure 5, right column). Importantly though, for all beam sizes, the KWW exponent is smaller than 1, proving that M3 yields slower-than-exponential correlation functions. We note that during our simulations we have observed that the exact value of the exponent is rather dependent on the fitting procedure and further and more extensive calculations are needed to clarify this issue. Some of the discrepancies may disappear by averaging over many realisations (i.e., repeating many simulations and averaging them), but, nonetheless, a more exhaustive study combining simulations and theory is still required and will be reported elsewhere. 

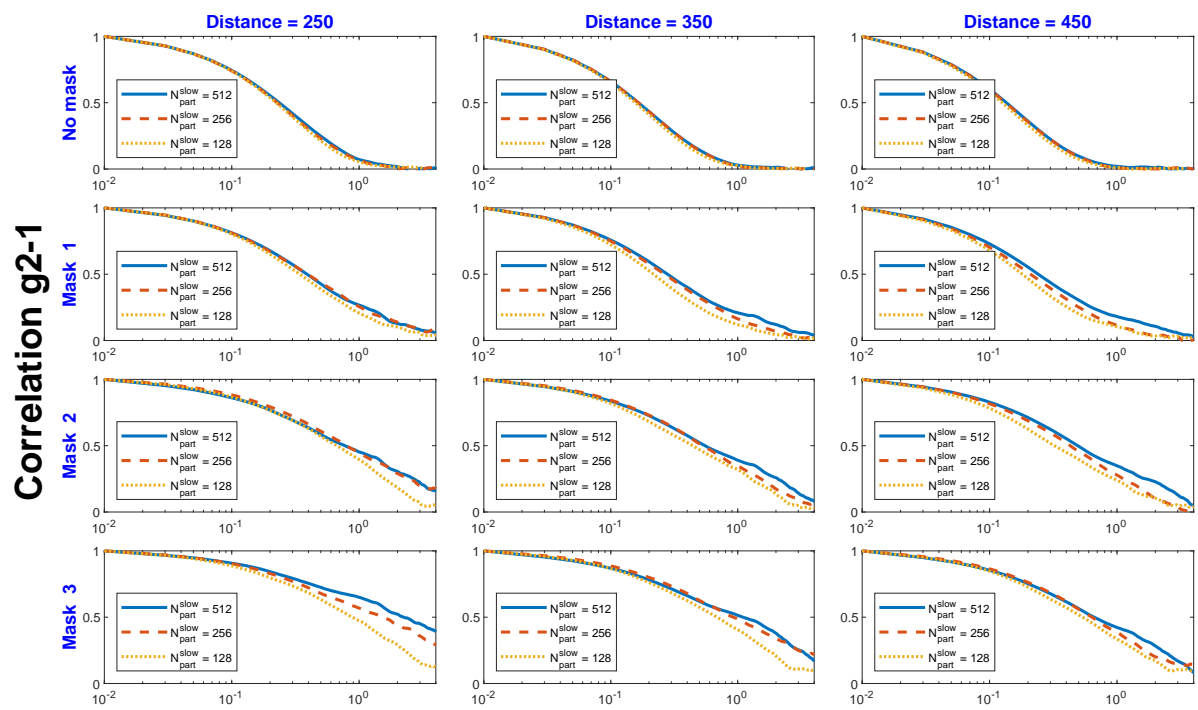

Time [arb. units]

Figure 4. One-time correlation functions obtained with model 3 and three different concentrations (i.e., number of slow particles, $N_{2}$ ) in the confined area $A_{2}$, at momentum transfer distances 250,350 and 450 , and for different masks.
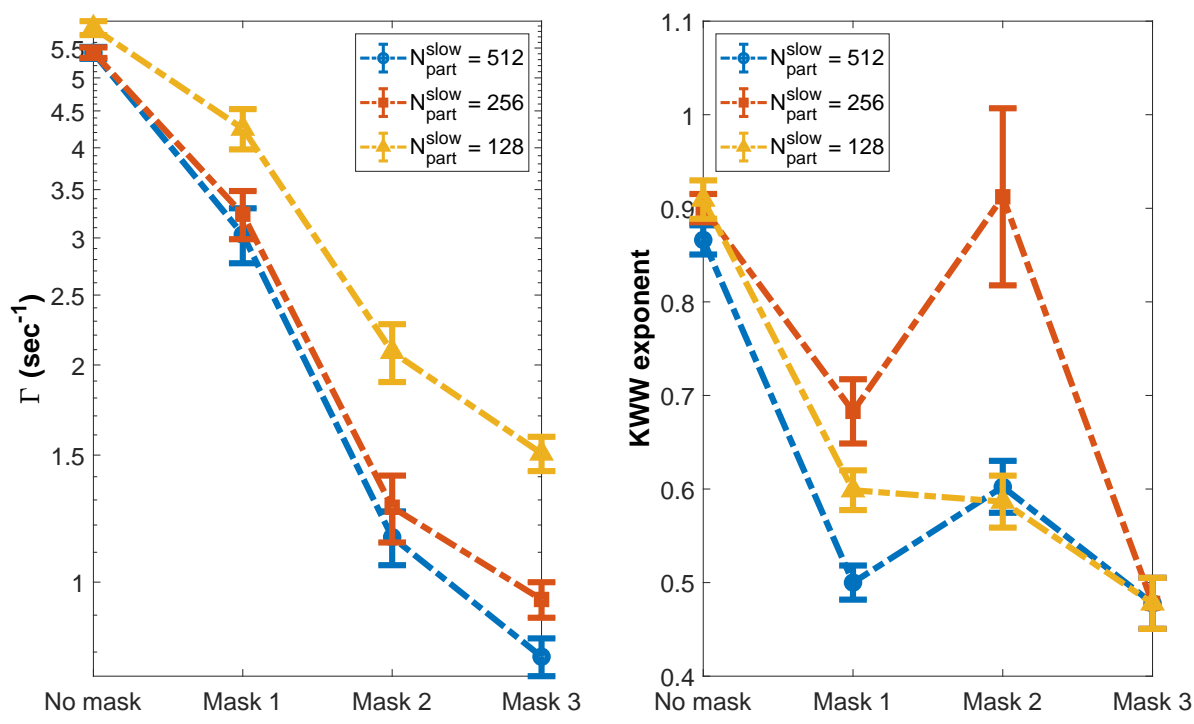

Figure 5. Decay rate $(\Gamma)$ (left) and KWW exponents (right) for different masks and slow moving particle concentration in model 3. The values have been extracted fitting the OTCFs of Figure 4 with a KWW function (see text for details).

\subsection{Raster Scanning}

As a further practical example, we simulate a system with a lateral variation of dynamics that could represent a liquid in confinement-although the dynamics we have employed, namely Brownian motion, are professedly not correct for a liquid-and show that a small illumination can be combined with a "raster" scan approach, in a similar way as is employed in scanning imaging approaches to obtain a spatial map of the system dynamics with a resolution only limited by the beam size. The system (Model 4, M4) is schematically presented in Figure 6 (left). All particles in the system follow Brownian motion but with different diffusion constants (numerical parameters can be found in Table 2). We have separated the system in five areas: the two areas close to the left and right borders have the slowest diffusion constant. The central area contains the fastest particles and the particles in the band bounded by the border and central bands have a diffusion constant in between. We have 
evaluated the OTCFs using a beam illuminating the whole system (no mask), and using a small beam illuminating each band. The results are shown in Figure 6 (right).

Table 2. Simulation parameters for model 4. Particle types in the top row and related parameters in the rows below with: $N$ their respective number, $A$ the initial area (in pixels) on which their are positioned and $\eta$ their diffusion constant.

\begin{tabular}{cccc}
\hline Particles & FAST & MEDIUM & SLOW \\
\hline$N$ & 1024 & 512 & 1024 \\
$A$ & $320 \times 1024$ & $196 \times 1024$ & $160 \times 1024$ \\
$\eta$ & $3 \eta_{\text {slow }}$ & $2 \eta_{\text {slow }}$ & 64 \\
mask & & $101 \times 101$ & \\
\hline
\end{tabular}

When the beam is rastered from left to right, from the region with slow particles towards the region richer in fast particles, the OTCF decay accelerates until it reaches it maximum decay rate in the central part of M4. Past the fast region and going towards the medium and slow motion regions, the OTCFs' decay slows down again. If the system were illuminated fully, an "average" OTCF with a decay rate amidst the fastest and the slowest one is obtained (yellow line on the right inset of Figure 6). Thus, a small beam does indeed allow for exploring and mapping the various dynamics on the model.
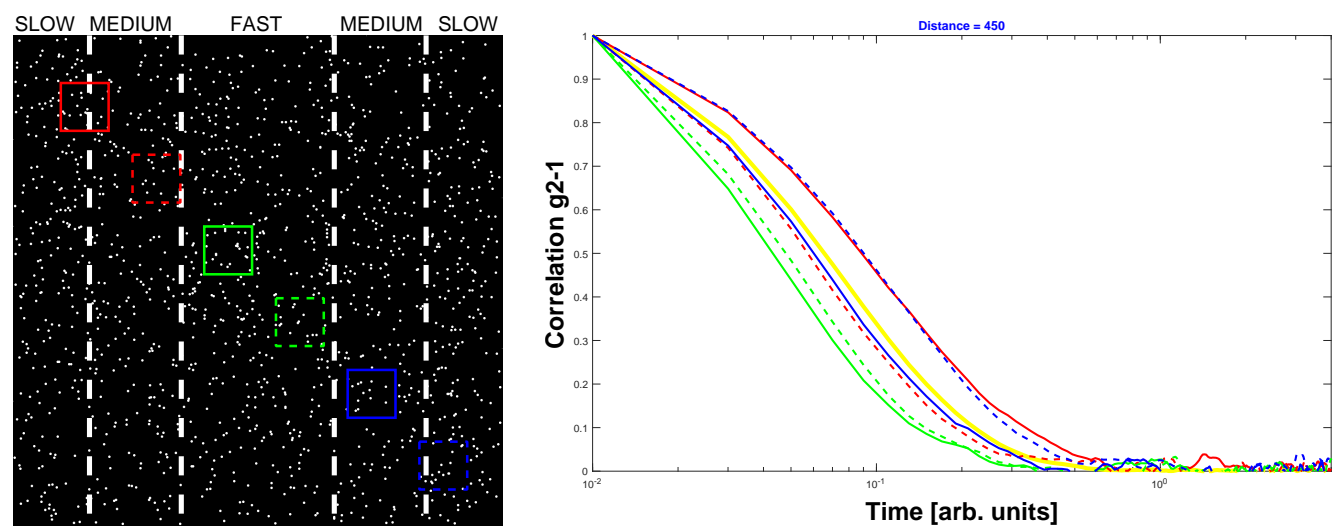

Figure 6. Schematic representation of model 4 (left) and OTCFs extracted rastering the beam along the diagonal (right). The squares indicate the position at which the OTCFs have been extracted. The corresponding OTCs follow the same colour and line style (solid or dashed). The yellow solid line corresponds to the OTCF extracted using the full beam (i.e., illuminating the whole system).

\section{Discussion}

The study presented here is purely speculative: our analysis is performed using synthetic data on a model system, and therefore many important experimental implications are not taken into account and should be discussed separately. While in principle rather easy to perform, XPCS measurements are experimentally terribly demanding. The stability required for the setup will scale with both the beam size and the minimum acquisition time. Another important aspect to consider is that the dramatic increase of flux density has a devastating effect for systems that are sensitive to beam damage. Nonetheless, focusing of X-rays with the aim to increase the available coherent flux has already been used (see for example [46], and [7] for more references), while no investigation of systems with high lateral resolution, as we propose, has been reported yet. Test experiments attempted until now by the authors using $100 \mathrm{~nm}$ beam size (not shown here) have been strongly limited by the instability of the available setups and the readout time of the used detectors.

Beside the archetypal exponentially decaying OTCFs which often arise in stochastic processes, there are many dynamic systems that display other functional forms of OTCFs (see [47] for a particularly relevant discussion). In most cases, these deviations result from the intrinsic dynamics 
or dynamical heterogeneities. Accessing dynamics with spatial resolution is especially appropriate to study heterogeneous systems. A spatially homogeneous system where all local contributions are equal to the ensemble averaged ones and a spatially heterogeneous system with locally different dynamics can indeed present identical OTCFs if only macroscale or ensemble averaged (i.e., large beam) information is extracted [48]. Our results in Section 4.1 show that a beam size comparable to the heterogeneity size brings in new information that is generally concealed with a large beam. The caveat is that this new information is not necessarily related in a straightforward way to the dynamics of the heterogeneous part as different contributions can still concur even when only very small areas are probed. However, for those instances too, collecting OTCFs at different areas on a sample may turn essential to delimit the time scales present on the sample and construct reliable models to analyze the data.

Using nanobeams is probably the most direct method to get information about the different dynamics in heterogeneous systems but other approaches have been developed as well that we report here for completeness. Andrews et al. [49] have nicely demonstrated that alternative ways used to extract the different time scales of heterogeneous systems from DLS data can be extended and adapted for XPCS too. Nevertheless, those methods will give information over the whole illuminated volume and thus ensemble averaged and lacking the higher spatial resolution nanobeams provide. That is, for the model introduced in Section 4.3 and applying such methods to the OTCF extracted using the full beam (yellow solid line in Figure 6), one may determine the different timescales that contribute to it (red, blue and green curves in Figure 6), but not which area of the sample they come from. For many cases, that approach will suffice and may even be combined with data acquired with nanobeams. The spatial distribution can be determined with raster scans.

Heterogeneous systems with nanoscale heterogeneities being ubiquitous, the number of studies that could benefit from nXPCS is immense: liquids, glasses, self-organised systems, oscillatory chemical reactions, magnetic spin ices, polymers or colloids in confinement, nanofluidics. To mention only two types: (a) Similar studies to the one reported by Zhang et al. [50] on the spatially heterogeneous dynamics in metallic glass forming systems can be envisaged, not with the spectacularly high spatial resolution reported in that work, which has been obtained using electrons as probe, but on more complex systems or environments that are not accessible with electron probes; (b) Using a sufficiently small beam, it will be possible to probe the inter-island (or collective) and intra-island magnetic reversal dynamics in artificial spin ice structures or magnetic domains [28-30,51]. Morley et al. have studied square arrays of $30 \times 70 \mathrm{~nm}$ size magnetic islands, using a $10 \mu \mathrm{m}$ diameter beam, at $9.6^{\circ}$ incidence angle [30]. The $>6000$ islands thus illuminated provide information about the collective dynamics of the spin ice fluctuating magnetic configurations. A focused $\sim 100 \mathrm{~nm}$ beam with a flux density 1000-to-80,000 times larger could provide information about the spin fluctuations of fewer, or even single, islands at similar time-scales (in this case, hundreds of seconds). This statement manifestly passes over all the inherent technical difficulties arising from stability or sample damage. Specific preparation and calculations are needed for each experiment to determine its feasibility. If studies on individual islands are viable, then they could provide valuable local information about the dynamics of tailored two-dimensional nano-magnetic systems [52,53].

Most XPCS studies on surfaces [4] would benefit from a smaller beam. Surface studies are performed with a beam impinging at very low angles. The beam projection onto the sample depends on the incidence angle as $\alpha$ as $S_{v} / \sin \alpha$, where $S_{v}$ is the vertical size of the beam. By using strong focusing along the vertical direction, the footprint on the sample surface can be made comparable to the sample length in the beam direction, increasing the efficiency of the measurement. In addition, given the importance that the surface morphology (kinks, steps, terraces...) has on surface properties such as diffusion, reactivity, etc. [54] studies such as those reported in $[55,56]$ may also profit from reduced illumination.

As shrinking the beam results in larger speckles, focusing can incidentally be used to optimise the sample-to-detector distance too [43]. Another feature of focused beams that can be exploited is 
the variation of their size along the beam propagation direction. nXPCS measurements can thus be performed by placing the sample at different distances from the focal plane to change the beam footprint on the sample and at the same time control the flux density on the sample. This can be extremely useful to determine conditions for beam damage, or intrinsic physical behaviours, like determining if it is the total flux or rather the flux density the factor controlling the pumping of atoms observed using X-rays [57]. The high flux density nanobeams can provide, and which may potentially lead to beam damage, will surely restrict nXPCS to samples not prone to radiation damage.

An extremely important point to remark is that, for $n X P C S$, the rather direct connection between the intermediate scattering function and the intensity correlation function given by Siegert's formula (Equation (2)) has to be dropped out. One of necessary assumptions for the Siegert relation to be valid is that the coherence volume contains a large number of independent scatterers [58] but that condition is not necessarily met when using nanobeams. Voigt and Hess have distinctly shown that deviations from the Siegert relation are caused by number fluctuations or local ordering [34]. The correct interpretation and extraction of physical parameters from the intensity correlation functions in nXPCS experiments will require numerical simulations. This is not necessarily a drawback because the illuminated volume is small and in certain cases simulations with a small number of particles will be sufficient. However, for systems that use 'mesoscopic' real space simulations to describe their properties, very large matrices (or a high density of points in real space) may be necessary to extract portions of these simulations with enough number of points and calculate the expected scattered signal when using a nanobeam. Ion eroded surface or growth simulations are a good example of such systems [59-61], and have also been investigated with conventional XPCS [19,20,61]. Simulations of real systems must undoubtedly go beyond archetypal simulations. In most cases, full 3D simulations that take into account the parameters controlling the intrinsic dynamics will be needed. The scattering factors of the material under study and the X-ray energy, especially for resonant XPCS studies [28,30], must be included. A marked difference with conventional XPCS is that, in nXPCS, the beam has a particular intensity distribution and this should be built into the simulations too. In conventional XPCS, one can consider that the intensity is constant across the whole beam but coherent nanobeams have a more complex beam profile (see, for example, [62,63]). Gaussian beam profiles can be approximated using analytic formulas, as it has already been done in DLS [58]. The beam waist and depth of focus along the direction of beam propagation are also an important factor because, for thick samples (thicker than the depth of focus), the effective illumination may be larger than the beam size at the focus.

Combining XPCS with nanobeams just starts to be realistic. We have performed a first experimental trial at the NanoMAX beamline of MAXIV during the early phase of the beamline commissioning. We used a $100 \mathrm{~nm}$ size beam with $0.09^{\circ}$ divergence. The nanobeam was obtained using KB mirror optics, with $\sim 180 \mathrm{~mm}$ focal length [63]. The flux at the sample was $\sim 3 \times 10^{9} \mathrm{ph} / \mathrm{s}$. Using a Pilatus $1 \mathrm{M}$ (pixel size: $175 \times 175 \mu \mathrm{m}^{2}$ at $4.2 \mathrm{~m}$ from the sample, we could observe time-evolving speckles from a $\mathrm{SiO}_{2}$ sample [64]. However, those changes of the speckle patterns we observed in our test experiments were not fully due to the system dynamics [57], but to setup instabilities that hampered nXPCS measurements as evidenced from the analysis of the two-time correlation functions from a static system. Despite this temporary defeat, we are very confident that soon nXPCS will become realizable.

Author Contributions: Both authors have contributed equally though not identically to this work. In more detail: Conceptualization, O.B. and D.C.; Formal analysis \& Software O.B.; Methodology, O.B. and D.C.; Visualisation O.B., Writing original draft, O.B. and D.C. All authors have read and agreed to the published version of the manuscript.

Funding: O.B. gratefully acknowledges the financial support of the UK Engineering and Physical Sciences Research Council (EPSRC).

Conflicts of Interest: The authors declare no conflict of interest. The funders had no role in the design of the study; in the collection, analyses, or interpretation of data; in the writing of the manuscript, or in the decision to publish the results. 


\section{Abbreviations}

The following abbreviations are used in this manuscript:

$\begin{array}{ll}\text { CCD } & \text { Charge-coupled device } \\ \text { DLS } & \text { Dynamic light scattering } \\ \text { DLSR } & \text { Diffraction limited storage rings } \\ \text { FEL } & \text { Free electron laser } \\ \text { KB } & \text { Kirkpatrick-Baez } \\ \text { KKW } & \text { Kohlrausch-Williams-Watts } \\ \text { M1, M2, M3 and M4 } & \text { Models 1, 2, 3 and 4 } \\ \text { nXPCS } & \text { nano X-ray photon correlation spectroscopy } \\ \text { OTCF } & \text { One-time correlation function } \\ \text { XPCS } & \text { X-ray photon correlation spectroscopy }\end{array}$

\section{References}

1. Eriksson, M.; van der Veen, J.F.; Quitmann, C. Diffraction-limited storage rings? A window to the science of tomorrow. J. Synchrotron Radiat. 2014, 21, 837-842. [CrossRef]

2. Grübel, G.; Madsen, A.; Robert, A. X-ray Photon Correlation Spectroscopy (XPCS). In Soft Matter Characterization; Borsali, R., Pecora, R., Eds.; Springer: Berlin, Germany, 2008; pp. 953-995.

3. Sutton, M. A review of X-ray intensity fluctuation spectroscopy. C. R. Phys. 2008, 9, 657-667. [CrossRef]

4. Sinha, S.K.; Jiang, Z.; Lurio, L.B. X-ray Photon Correlation Spectroscopy Studies of Surfaces and Thin Films. Adv. Mater. 2014, 26, 7764-7785. [CrossRef] [PubMed]

5. Gutt, C.; Sprung, M. X-ray Photon Correlation Spectroscopy. In X-ray Diffraction: Modern Experimental Techniques; Seeck, O.H., Murphy, B.M., Eds.; CRC Press: Singapore, 2015; pp. 385-419.

6. Madsen, A.; Fluerasu, A.; Ruta, B. Structural Dynamics of Materials Probed by X-ray Photon Correlation Spectroscopy. In Synchrotron Radiation and Free-Electron Lasers; Jaeschke, E., Khan, S., Schneider, J.R., Hastings, J.B., Eds.; Springer: Berlin, Germany, 2015; pp. 1617-1641.

7. Bikondoa, O. X-ray photon correlation spectroscopy for the characterization of soft and hard condensed matter. In X-ray and Neutron Techniques for Nanomaterials Characterization; Kumar, C.S.S.R., Ed.; Springer: Berlin, Germany, 2016; pp. 95-156.

8. Zhang, Q.; Dufresne, E.M.; Sandy, A.S. Dynamics in hard condensed matter probed by X-ray photon correlation spectroscopy: Present and beyond. Curr. Opin. Solid State Mater. Sci. 2018, 22, 202-212. [CrossRef]

9. Sandy, A.R.; Zhang, Q.; Lurio, L.B. Hard X-ray Photon Correlation Spectroscopy Methods for Materials Studies. Annu. Rev. Mater. Res. 2018, 48, 9.1-9.24. [CrossRef]

10. Zhang, Q.; Dufresne, E.M.; Narayanan, S.; Maj, P.; Koziol, A.; Szczygiel, R.; Grybos, P.; Sutton, M.; Sandy, A.R. Sub-microsecond-resolved multi-speckle $\mathrm{X}$-ray photon correlation spectroscopy with a pixel array detector. J. Synchrotron Radiat. 2018, 25, 1408-1416. [CrossRef]

11. Seaberg, M.H.; Holladay, B.; Lee, J.C.T.; Sikorski, M.; Reid, A.H.; Montoya, S.A.; Dakovski, G.L.; Koralek, J.D.; Coslovich, G.; Moeller, S.; et al. Nanosecond X-Ray Photon Correlation Spectroscopy on Magnetic Skyrmions. Phys. Rev. Lett. 2017, 119, 067403. [CrossRef]

12. Berne, B.J.; Pecora, R. Dynamic Light Scattering; Dover: New York, NY, USA, 2000.

13. Pusey, P.N. Dynamic Light Scattering. In Neutrons X-rays and Light: Scattering Methods Applied to Soft Condensed Matter; Lindner, P., Zemb, T., Eds.; Elsevier North Holland: Amsterdam, The Netherlands, 2002; pp. 203-220.

14. Chu, B. Laser Light Scattering; Dover: New York, NY, USA, 2007.

15. Hoshino, T.; Tanaka, Y.; Jinnai, H.; Takahara, A. Surface and Interface Analyses of Polymer Brushes by Synchrotron Radiation. J. Phys. Soc. Jpn. 2013, 82, 021014. [CrossRef]

16. Shpyrko, O.G. X-ray photon correlation spectroscopy. J. Synch. Rad. 2014, 21, 1057-1064. [CrossRef]

17. Fluerasu, A.; Kwasniewski, P.; Caronna, C.; Destremaut, F.; Salmon, J.B.; Madsen, A. Dynamics and rheology under continuous shear flow studied by X-ray photon correlation spectroscopy. New J. Phys. 2010, 12, 035023. [CrossRef]

18. Leheny, R.L. XPCS: Nanoscale motion and rheology. Curr. Opin. Colloid Interface Sci. 2012, 17, 3-12. [CrossRef] 
19. Bikondoa, O.; Carbone, D.; Chamard, V.; Metzger, T.-H. Ageing dynamics of ion erosion induced self-organization processes. Sci. Rep. 2013, 3, 1850. [CrossRef]

20. Mokhtarzadeh, M.; Ulbrandt, J.G.; Myint, P.; Narayanan, S.; Headrick, R.L.; Ludwig, K.F., Jr. Nanoscale kinetics and dynamics during $\mathrm{Ar}^{+}$patterning of $\mathrm{SiO}_{2}$. Phys. Rev. B 2019, 99, 165429. [CrossRef]

21. Ruta, B.; Baldi, G.; Monaco, G.; Chushkin, Y. Compressed correlation functions and fast aging dynamics in metallic glasses. J. Chem. Phys. 2013, 138, 054508. [CrossRef] [PubMed]

22. Ludwig, K.F., Jr.; Livet, F.; Bley, F.; Simon, J.-P.; Caudron, R.; Le Bolloc'h, D.; Moussaid, A. X-ray intensity fluctuation spectroscopy studies of ordering kinetics in a Cu-Pd alloy. Phys. Rev. B 2001, 72, 144201. [CrossRef]

23. Livet, F.; Bley, F.; Caudron, R.; Geissler, E.; Abernathy, D.; Detlefs, C.; Grübel, G.; Sutton, M. Kinetic evolution of unmixing in an AlLi alloy using X-ray intensity fluctuation spectroscopy. Phys. Rev. E 2001, 63, 036108. [CrossRef]

24. Leitner, M.; Sepiol, B.; Stadler, L.M.; Pfau, B.; Vogl, G. Atomic diffusion studied with coherent X-rays. Nat. Mater. 2009, 8, 717-720. [CrossRef]

25. Fluerasu, A.; Sutton, M.; Dufresne, E.M. X-ray intensity fluctuation spectroscopy studies on phase-ordering systems. Phys. Rev. Lett. 2005, 94, 055501. [CrossRef]

26. Pierce, M.S.; Hennessy, D.C.; Chang, K.C.; Komanicky, V.; Strzalka, J.; Sandy, A.; Barbour, A.; You, H. Persistent oscillations of X-ray speckles: Pt (001) step flow. Appl. Phys. Lett. 2011, 99, 121910. [CrossRef]

27. Shpyrko, O.G.; Isaacs, E.D.; Logan, J.M.; Feng, Y.; Aeppli, G.; Jaramillo, R.; Kim, H.C.; Rosenbaum, T.F.; Zschack, P.; Sprung, M.; et al. Direct measurement of antiferromagnetic domain? Nature 2007, 447, 68-71. [CrossRef]

28. Chen, X.M.; Farmer, B.; Woods, J.S.; Dhuey, S.; Hu, W.; Mazzoli, C.; Wilkins, S.B.; Chopdekar, R.V.; Scholl, A.; Robinson, I.K.; et al. Spontaneous Magnetic Superdomain Wall Fluctuations in an Artificial Antiferromagnet. Phys. Rev. Lett. 2019, 123, 197202. [CrossRef] [PubMed]

29. Chesnel, K.; Nelson, J.A.; Kevan, S.D.; Carey, M.J.; Fullerton, E.E. Oscillating spatial dependence of domain memory in ferromagnetic films mapped via X-ray speckle correlation. Phys. Rev. B 2011, 83, 054436. [CrossRef]

30. Morley, S.A.; Alba Venero, D.; Porro, J.M.; Riley, S.T.; Stein, A.; Steadman, P.; Stamps, R.L.; Langridge, S.; Marrows, C.H. Vogel-Fulcher-Tammann freezing of a thermally fluctuating artificial spin ice probed by $\mathrm{X}$-ray photon correlation spectroscopy. Phys. Rev. B 2017, 95, 104422. [CrossRef]

31. Zhang, Q.; Dufresne, E.M.; Chen, P.; Park, J.; Cosgriff, M.P.; Yusuf, M.; Dong, Y.; Fong, D.D.; Zhou, H.; Cai, Z.; et al. Thermal Fluctuations of Ferroelectric Nanodomains in a Ferroelectric-Dielectric $\mathrm{PbTiO}_{3} / \mathrm{SrTiO}_{3}$ Superlattice. Phys. Rev. Lett. 2018, 118, 097601. [CrossRef]

32. Jacques, V.L.R.; Laulhè, C.; Moisan, N.; Ravy, S.; Le Bolloc'h, D. Laser-Induced Charge-Density-Wave Transient Depinning in Chromium. Phys. Rev. Lett. 2016, 117, 156401. [CrossRef]

33. Alonso-Mori, R.; Caronna, C.; Chollet, M.; Curtis, R.; Damiani, D.S.; Defever, J.; Feng, Y.; Flath, D.L.; Glownia, J.M.; Lee, S.; et al. The X-ray Correlation Spectroscopy instrument at the Linac Coherent Light Source. J. Synchrotron Radiat. 2015, 22, 508-513. [CrossRef]

34. Voigt, H.; Hess, S. Comparison of the intensity correlation function and the intermediate scattering function of fluids: A molecular dynamics study of the Siegert relation. Phys. A 1994, 202, 145-164. [CrossRef]

35. Dainty, J.C. (Ed.) Laser Speckle, 2nd ed.; Springer: Berlin, Germany, 1984.

36. Loudon, R. The Quantum Theory of Light; Oxford University Press: Oxford, UK, 1983.

37. Jakeman, E. Photon Correlation. In Photon Correlation and Light Beating Spectroscopy; Cummins, H.Z., Pike, E.R., Eds.; Plenum Press: New York, NY, USA, 1974; pp. 75-149.

38. Madsen, A.; Leheny, R.L.; Guo, H.; Sprung, M.; Czakkel, O. Beyond simple exponential correlation functions and equilibrium dynamics in X-ray photon correlation spectroscopy. New J. Phys. 2010, 12, 055001-055016. [CrossRef]

39. Chaikin, P.M.; Lubensky, T.C. Principles of Condensed Matter Physics; Cambridge University Press: Cambridge, UK, 1995.

40. Mandel, L.; Wolf, E. Optical Coherence and Quantum Optics; Cambridge University Press: Cambridge, UK, 1995.

41. Falus, P.; Borthwick, M.A.; Mochrie, S.G.J. Fast CCD camera for X-ray photon correlation spectroscopy and time-resolved X-ray scattering and imaging. Rev. Sci. Instrum. 2004, 75, 4383. [CrossRef] 
42. Zinn, T.; Homs, A.; Sharpnack, L.; Tinti, G.; Fröjdh, E.; Douissard, P.-A.; Kocsis, M.; Möller, J.; Chushkin, Y.; Narayanan, T. Ultra-small-angle X-ray photon correlation spectroscopy using the Eiger detector. J. Synchrotron Radiat. 2018, 25, 1753-1759. [CrossRef]

43. Falus, P.; Lurio, L.B.; Mochrie, S.G.J. Optimizing the signal-to-noise ratio for X-ray photon correlation spectroscopy. J. Synchrotron Radiat. 2006, 13, 253-259. [CrossRef] [PubMed]

44. Available online: https://www.mathworks.com/products/matlab.html (accessed on 28 August 2020).

45. Burkardt, J. Brownian Motion Simulation. Available online: https://people.sc.fsu.edu/ jburkardt/m_src/ brownian_motion_simulation/brownian_motion_simulation.html (accessed on 28 August 2020).

46. Sandy, A.R.; Narayanan, S.; Sprung, M.; Su, J.-D.; Evans-Lutterodt, K.; Isakovic, A.F.; Stein, A. Kinoform optics applied to X-ray photon correlation spectroscopy. J. Synchrotron Radiat. 2010, 17, 314-320. [CrossRef] [PubMed]

47. Pecora, R. Basic concepts-Scattering and time correlation functions. In Soft Matter Characterization; Borsali, R., Pecora, R., Eds.; Springer: Berlin, Germany, 2008; pp. 1-40.

48. Richert, R. Heterogeneous dynamics in liquids: Fluctuations in space and time. J. Phys. Condens. Matter 2002, 14, R703-R738. [CrossRef]

49. Andrews, R.N.; Narayanan, S.; Zhang, F.; Kuzmenko, I.; Ilavsky, J. Inverse transformation: Unleashing spatially heterogeneous dynamics with an alternative approach to XPCS data analysis. J. Appl. Cryst. 2018, 51, 35-46. [CrossRef]

50. Zhang, P.; Maldonis, J.J.; Liu, Z.; Schroers, J.; Voyles, P.M. Spatially heterogeneous dynamics in a metallic glass forming liquid imaged by electron correlation microscopy. Nat. Commun. 2018, 9, 1129. [CrossRef]

51. Gorfman, S.; Bokov, A.A.; Davtyand, A.; Reiser, M.; Xie, Y.; Yeb, Z.-G.; Zozulya, A.V.; Sprung, M.; Pietsch, U.; Gutt, C. Ferroelectric domain wall dynamics characterized with X-ray photon correlation spectroscopy. Proc. Natl. Acad. Sci. USA 2018, 115, E6680-E6689. [CrossRef]

52. Östman, E.; Arnalds, U.B.; Kaplakis, V.; Taroni, A.; Hjörvarsson, B. Ising-like behaviour of mesoscopic magnetic chains. J. Phys. Condens. Matter 2018, 30, 365301. [CrossRef]

53. Östman, E.; Stopfel, H.; Chioar, I.-A.; Arnalds, U.B.; Stein, A.; Kaplakis, V.; Hjörvarsson, B. Interaction modifiers in artificial spin ices. Nat. Phys. 2018, 14, 375-379. [CrossRef]

54. Ertl, G.; Küppers, J. Low Energy Electrons and Surface Chemistry; VCH Verlagsgesellschaft: Weinheim, Germany, 1985.

55. Ulbrandt, J.G.; Rainville, M.G.; Wagenbach, C.; Narayanan, S.; Sandy, A.R.; Zhou, H.; Ludwig, K.F., Jr.; Headrick, R.L. Direct measurement of the propagation velocity of defects using coherent X-rays. Nat. Phys. 2016, 12, 794-799. [CrossRef]

56. Headrick, L.; Ulbrandt, J.G.; Myint, P.; Wan, J.; Li, Y.; Fluerasu, A.; Zhang, Y.; Wiegart, L.; Ludwig, K.F., Jr. Coherent $\mathrm{X}$-ray measurement of step-flow propagation during growth on polycrystalline thin film surfaces. Nat. Commun. 2019, 10, 2638. [CrossRef]

57. Ruta, B.; Zontone, F.; Chushkin, Y.; Baldi, G.; Pintor, G.; Monaco, G.; Rufflé, B.; Kob, W. Hard X-rays as Pump Probe At. Motion Oxide Glasses. Sci. Rep. 2017, 7, 3962. [CrossRef] [PubMed]

58. Pusey, P.N. Statistical Properties of Scattered Radiation. In Photon Correlation Spectroscopy and Velocimetry; Cummins, H.Z., Pike, E.R., Eds.; Plenum Press: New York, NY, USA, 1977; pp. 45-141.

59. Bikondoa, O.; Carbone, D.; Chamard, V.; Metzger, T.H. Ion beam sputtered surface dynamics investigated with two-time correlation functions: A model study. J. Phys. Condens. Matter 2012, 24, 445006. [CrossRef]

60. Mokhtarzadeh, M.; Ludwig, K.F., Jr. Simulations of Co-GISAXS during kinetic roughening of growth surfaces. J. Synchrotron Radiat. 2017, 24, 1187-1194. [CrossRef] [PubMed]

61. Ju, G.; Xu, D.; Highland, M.J.; Thompson, C.; Zhou, H.; Eastman, J.A.; Fuoss, P.H.; Zapol, P.; Kim, H.; Stephenson, G.B. Coherent X-ray spectroscopy reveals the persistence of island arrangements during layer-by-layer growth. Nat. Phys. 2019, 15, 589-594. [CrossRef]

62. Mastropietro, F.; Carbone, D.; Díaz, A.; Eymery, J.; Sentenac, A.; Metzger, T.H.; Chamard, V.; Favre-Nicolin, V. Coherent $X$-ray wavefront reconstruction of a partially illuminated Fresnel zone plate. Opt. Express 2011, 19, 19223-19232. [CrossRef] [PubMed] 
63. Björling, A.; Kalbfleisch, S.; Kahnt, M.; Sala, S.; Parfeniukas, K.; Vogt, U.; Carbone, D.; Johansson, U. Ptychographic characterization of a coherent nanofocused X-ray beam. Opt. Express 2020, 28, 5069-5078.

64. Bikondoa, O.; Carbone, D. Materiaren dinamikaren azterketa X izpi nanometrikoak eta fotoi korrelazioespektroskopia erabiliz. In Bai, Jauna, Bai: Fisika Euskaraz! Jose Ramon Etxebarria Irakaslearen Omenez; Bilbao, G., Gartzia, P., Menika, M.K., Eds.; UEU-Udako Euskal Unibertsitatea: Bilbo, Euskal Herria, 2018; pp. 261-270.

(C) 2020 by the authors. Licensee MDPI, Basel, Switzerland. This article is an open access article distributed under the terms and conditions of the Creative Commons Attribution (CC BY) license (http:/ / creativecommons.org/licenses/by/4.0/). 